\title{
Some Problems Concerning with Sasaki Metric on the Second-Order Tangent Bundles $\ddagger$
}

\author{
Abdullah Mağden, Aydın Gezer* and Kübra Karaca \\ (Communicated by Levent Kula)
}

\begin{abstract}
In this paper, we consider a second-order tangent bundle equipped with Sasaki metric over a Riemannian manifold. All forms of curvature tensor fields are computed. We obtained the relation between the scalar curvature of the base manifold and the scalar curvature of the second-order tangent bundle and presented some geometric results concerning with kinds of curvature tensor fields. Also, we search the weakly symmetry property of the second-order tangent bundle. Finally, we end our paper with statistical structures on the second-order tangent bundle.
\end{abstract}

\section{Introduction}

The second-order tangent bundle $T^{2} M$ over an $n$-dimensional manifold $M$ was constructed in [15] by Yano and Ishihara. Also, they defined the lifts of tensor fields and connections given on $M$ to $T^{2} M$. Later, in [7], Dodson and Radivoiovici proved that a second-order tangent bundle $T^{2} M$ becomes a vector bundle over $M$ if and only if $M$ has a linear connection. The Sasaki metric $\widetilde{g}$ on the second-order tangent bundle $T^{2} M$ over a Riemannian manifold $(M, g)$ was firstly considered by Ishikawa and studied some properties by local coordinate in [10]. This metric also studied by some authors. We refer to [5, 7, 8, 9, 3]. In [8], the first and second authors constructed certain Riemannian almost product structures on $T^{2} M$ with Sasaki metric $\widetilde{g}$ and also presented some results concerning these structures. They locally calculated all forms of the curvature tensors on $T^{2} M$ and gave some local results. Also, they presented some geometrical properties of two metric connections with non-vanishing torsion on $\left(T^{2} M, \widetilde{g}\right)$. In this paper, we study some problems concerning with the second-order tangent bundle $T^{2} M$ with the Sasaki metric $\widetilde{g}$ and obtain the global results. $C^{\infty}$.

We point out here and once that all geometric objects considered in this paper are supposed to be of class

\section{Basic notations and definitions}

Let $(M, g)$ be an $n$-dimensional Riemannian manifold with the Levi-Civita connection $\nabla$ and $T^{2} M$ be its second-order tangent bundle. $T^{2} M$ is the set of all of 2 -jets at $0 \in \mathbb{R}$ of differentiable mappings $f: \mathbb{R} \longrightarrow M$ and is topologized. Then $T^{2} M$ is also an $3 n$-dimensional manifold. The tangent bundle $T M$ of $M$ is the set of all 1 -jets of $M$ and is a $2 n$-dimensional manifold. The canonical projection $\pi_{2}: T^{2} M \longrightarrow M$ defines the natural

Received : 14-04-2020, Accepted : 15-08-2020

* Corresponding author

₹ The paper is supported by the Scientific and Technological Research Council of Turkey, AR-GE 3001 Project No. 118F190. 
bundle structure of $T^{2} M$ over $M$. If we introduce the canonical projection $\pi_{12}: T^{2} M \longrightarrow T M$, then $T^{2} M$ has a bundle structure over the tangent bundle $T M$ with projection $\pi_{12}$.

Let $U$ be a coordinate neighbourhood of $M$ with $\left(x^{i}\right)$ as coordinates. The indices $i, j, k, \ldots$ take values $\{1,2, \ldots, n\}$. Hence, the system of local coordinates $\left(\pi_{2}^{-1}(U), x^{i}, u^{i}, z^{i}\right)$ is induced from a system of local coordinates $\left(U, x^{i}\right)$ in $M$. The coordinates $\left(x^{i}, u^{i}, z^{i}\right)$ in $\pi_{2}^{-1}(U)$ are called the induced coordinates. By putting

$$
\xi^{i}=x^{i}, \xi^{\bar{i}}=u^{i}, \xi^{\overline{\bar{i}}}=z^{i},
$$

we write the induced coordinates $\left(x^{i}, u^{i}, z^{i}\right)$ as $\left\{\xi^{A}\right\}$. The indices $A, B, C, \ldots$ run over the range $\{1,2, \ldots, n ; n+$ $1, n+2, \ldots, 2 n ; 2 n+1,2 n+2, \ldots, 3 n\}$.

Let $X=X^{i} \frac{\partial}{\partial x^{i}}$ be the local expression in $U$ of a vector field $X$ on $M$. Then the vector fields $X^{H_{0}}, X^{H_{1}}$ and $X^{H_{2}}$ on $T^{2} M$ are given, with respect to the induced coordinates $\left\{\xi^{A}\right\}$, by [3]

$$
\begin{gathered}
X^{H_{0}}=X^{j} \partial_{j}-u^{s} \Gamma_{s h}^{j} X^{h} \partial_{\bar{j}}-C_{h}^{j} X^{h} \partial_{\overline{\bar{j}}}, \\
X^{H_{1}}=X^{j} \partial_{\bar{j}}-2 u^{s} \Gamma_{s h}^{j} X^{h} \partial_{\overline{\bar{j}}}
\end{gathered}
$$

and

$$
X^{H_{2}}=X^{j} \partial_{\overline{\bar{j}}}
$$

with respect to the natural frame $\left\{\partial_{A}\right\}=\left\{\partial_{i}, \partial_{\bar{i}}, \partial_{\bar{i}}\right\}$ in $T^{2} M$, where $C_{h}^{j}=z^{m} \Gamma_{h m}^{j}+u^{s} u^{r}\left(\partial_{h} \Gamma_{s r}^{j}+\Gamma_{h m}^{j} \Gamma_{s r}^{m}-\right.$ $\left.2 \Gamma_{s m}^{j} \Gamma_{h r}^{m}\right), \Gamma_{s r}^{j}$ are the coefficients of the Levi-Civita connection $\nabla$ on $M$ and $\partial_{i}=\frac{\partial}{\partial x^{i}}, \partial_{\bar{i}}=\frac{\partial}{\partial u^{i}}, \partial_{\bar{i}}=\frac{\partial}{\partial z^{i}}$. For the Lie brackets of the vector fields $X^{H_{0}}, X^{H_{1}}$ and $X^{H_{2}}$ on $T^{2} M$, we have the following formulas:

$$
\left\{\begin{array}{l}
{\left[X^{H_{0}}, Y^{H_{0}}\right]=[X, Y]^{H_{0}}-(R(X, Y) u)^{H_{1}}-(R(X, Y) \omega)^{H_{2}},} \\
{\left[X^{H_{0}}, Y^{H_{1}}\right]=\left(\nabla_{X} Y\right)^{H_{1}},\left[X^{H_{0}}, Y^{H_{2}}\right]=\left(\nabla_{X} Y\right)^{H_{2}},} \\
{\left[X^{H_{a}}, Y^{H_{b}}\right]=0, a, b=1,2}
\end{array}\right.
$$

where $R$ is the Riemannian curvature tensor field of $\nabla$ on $M$ defined by $R(X, Y)=\left[\nabla_{X}, \nabla_{Y}\right]-\nabla_{[X, Y]}$ and where $\omega$ is locally expressed by $\omega^{i}=z^{i}+u^{s} u^{r} \Gamma_{s r}^{i}$ (for details, see [6]).

\section{Curvature properties of the Sasaki metric}

The Sasaki metric on the second-order tangent bundle $T^{2} M$ over a Riemannian manifold $(M, g)$ is defined by the identities:

$$
\begin{cases}\widetilde{g}\left(X^{H_{a}}, Y^{H_{b}}\right)=g(X, Y), & a=b \\ \widetilde{g}\left(X^{H_{a}}, Y^{H_{b}}\right)=0, & a \neq b\end{cases}
$$

for all vector fields $X, Y$ on $M$, where $a, b=0,1,2$.

For the Levi-Civita connection $\widetilde{\nabla}$ of $\widetilde{g}$ on $T^{2} M$, we have the following proposition.

Proposition 3.1. Let $(M, g)$ be an $n$-dimensional Riemannian manifold and $T^{2} M$ be its second-order tangent bundle. The Levi-Civita connection $\widetilde{\nabla}$ of $\widetilde{g}$ on $T^{2} M$ is given by the following conditions

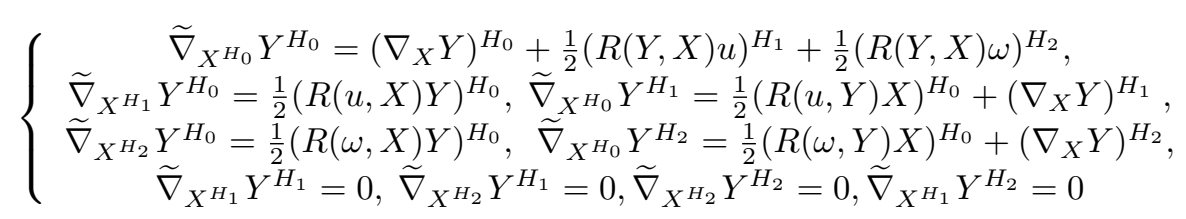

for all vector fields $X, Y$ on $M$ (see also [5]).

Let $F: T^{2} M \rightarrow T^{2} M$ be a smooth bundle endomorphism. Then we define the lifts of $F$ :

$$
\begin{aligned}
& F^{H_{0}}(u)=\sum u^{i} F\left(\partial_{i}\right)^{H_{0}}, F^{H_{1}}(u)=\sum u^{i} F\left(\partial_{i}\right)^{H_{1}}, F^{H_{2}}(u)=\sum u^{i} F\left(\partial_{i}\right)^{H_{2}}, \\
& F^{H_{0}}(\omega)=\sum \omega^{i} F\left(\partial_{i}\right)^{H_{0}}, F^{H_{1}}(\omega)=\sum \omega^{i} F\left(\partial_{i}\right)^{H_{1}}, F^{H_{2}}(\omega)=\sum \omega^{i} F\left(\partial_{i}\right)^{H_{2}} .
\end{aligned}
$$


Also, we obtain with the direct standard calculations

$$
\begin{aligned}
\widetilde{\nabla}_{X^{H_{0}}} u^{i} & =X^{H_{0}}\left(u^{i}\right)=-u^{s} \Gamma_{s h}^{i} X^{h}, \\
\widetilde{\nabla}_{X^{H_{1}}} u^{i} & =X^{i}, \widetilde{\nabla}_{X^{H_{2}}} u^{i}=0, \widetilde{\nabla}_{X^{H_{2}}} \omega^{i}=X^{i}, \\
\widetilde{\nabla}_{X^{H_{0}}} \omega^{i} & =-C_{h}^{i} X^{h}, \widetilde{\nabla}_{X^{H_{1}}} \omega^{i}=-2 u^{s} \Gamma_{s h}^{i} X^{h} .
\end{aligned}
$$

We shall now turn our attention to the Riemannian curvature tensor $\widetilde{R}$ of the Levi-Civita connection $\widetilde{\nabla}$ of $\widetilde{g}$ on $T^{2} M$. Firstly we give the following useful Lemma:

Lemma 3.2. Let $(M, g)$ be a Riemannian manifold and $\widetilde{\nabla}$ be the Levi-Civita connection of $\left(T^{2} M, \widetilde{g}\right)$. Let $F: T^{2} M \rightarrow$ $T^{2} M$ be a smooth bundle endomorphism, then

$$
\begin{aligned}
\widetilde{\nabla}_{X^{H_{0}}} F^{H_{0}}(u) & =\left(\nabla_{X} F(u)\right)^{H_{0}}+\frac{1}{2}(R(F(u), X) u)^{H_{1}}+\frac{1}{2}(R(F(u), X) \omega)^{H_{2}}, \\
\widetilde{\nabla}_{X^{H_{0}}} F^{H_{1}}(u) & =\frac{1}{2}(R(u, F(u)) X)^{H_{0}}+\left(\nabla_{X} F(u)\right)^{H_{1}}, \\
\widetilde{\nabla}_{X^{H_{0}}} F^{H_{2}}(u) & =\frac{1}{2}(R(\omega, F(u)) X)^{H_{0}}+\left(\nabla_{X} F(u)\right)^{H_{2}}, \\
\widetilde{\nabla}_{X^{H_{1}}} F^{H_{0}}(u) & =(F(X))^{H_{0}}+\frac{1}{2}(R(u, X) F(u)), \\
\widetilde{\nabla}_{X^{H_{1}}} F^{H_{1}}(u) & =(F(X))^{H_{1}}, \widetilde{\nabla}_{X^{H_{1}}} F^{H_{2}}(u)=(F(X))^{H_{2}}, \\
\widetilde{\nabla}_{X^{H_{2}}} F^{H_{0}}(u) & =0, \widetilde{\nabla}_{X^{H_{2}}} F^{H_{0}}(u)=0, \widetilde{\nabla}_{X^{H_{2}}} F^{H_{1}}(u)=0, \widetilde{\nabla}_{X^{H_{2}}} F^{H_{2}}(u)=0, \\
\widetilde{\nabla}_{X^{H_{0}}} F^{H_{0}}(\omega) & =\left(\nabla_{X} F(\omega)\right)^{H_{0}}+\frac{1}{2}(R(F(\omega), X) u)^{H_{1}}+\frac{1}{2}(R(F(\omega), X) \omega)^{H_{2}}, \\
\widetilde{\nabla}_{X^{H_{0}}} F^{H_{1}}(\omega) & =\frac{1}{2}\left(R(u, F(\omega) X)^{H_{0}}+\left(\nabla_{X} F(\omega)\right)^{H_{1}},\right. \\
\widetilde{\nabla}_{X^{H_{0}}} F^{H_{2}}(\omega) & =\frac{1}{2}\left(R(\omega, F(\omega) X)^{H_{0}}+\left(\nabla_{X} F(\omega)\right)^{H_{2}},\right. \\
\widetilde{\nabla}_{X^{H_{1}}} F^{H_{0}}(\omega) & =\frac{1}{2}(R(u, X) F(\omega))^{H_{0}}, \widetilde{\nabla}_{X^{H_{0}}} F^{H_{1}}(\omega)=0, \widetilde{\nabla}_{X^{H_{0}}} F^{H_{2}}(\omega)=0, \\
\widetilde{\nabla}_{X^{H_{2}}} F^{H_{0}}(\omega) & =(F(X))^{H_{0}}+\frac{1}{2}(R(\omega, X) F(\omega))^{H_{0}}, \\
\widetilde{\nabla}_{X^{H_{2}}} F^{H_{1}}(\omega) & =(F(X))^{H_{1}}, \widetilde{\nabla}_{X^{H_{2}}} F^{H_{2}}(\omega)=(F(X))^{H_{2}}
\end{aligned}
$$

for any vector field $X$ on $M$ and $u, \omega \in T^{2} M$.

In [5], Dida, Hathout and Djaa studied the geometry of the Sasaki metric $\widetilde{g}$ on $T^{2} M$. Their results are partially contained in Proposition 3.1 and Lemma 3.2. In their paper authors attempt to calculate the Riemannian curvature tensor field $\widetilde{R}$, the sectional curvature tensor field $\widetilde{K}$ and the scalar curvature $\widetilde{S}$ but unfortunately their calculations are wrong. They missed some parts of the Riemannian curvature tensor $\widetilde{R}$. In the rest of this section we shall correct the error and obtain valid expressions for $\widetilde{R}, \widetilde{K}$ and $\widetilde{S}$. Also note that local results for $T^{2} M$ with $\widetilde{g}$ were given in [8].

For Riemannian curvature tensor field $\widetilde{R}$ of the Levi-Civita connection $\widetilde{\nabla}$ of $\widetilde{g}$ on $T^{2} M$, we have

Proposition 3.3. Let $(M, g)$ be a Riemannian manifold and $T^{2} M$ be its second-order tangent bundle equipped with the Sasaki metric $\widetilde{g}$. The curvature tensor field $\widetilde{R}$ of the Levi-Civita connection $\widetilde{\nabla}$ of $\widetilde{g}$ on $T^{2} M$ is given by the following formulas:

$$
\begin{aligned}
& i) \widetilde{R}\left(X^{H_{0}}, Y^{H_{0}}\right) Z^{H_{0}} \\
& =\left\{\begin{array}{c}
R(X, Y) Z+\frac{1}{4}[R(u, R(Z, Y) u) X-R(u, R(Z, X) u) Y-2 R(u, R(Y, X) u) Z] \\
+\frac{1}{4}[R(\omega, R(Z, Y) \omega) X-R(\omega, R(Z, X) \omega) Y-2 R(\omega, R(Y, X) \omega) Z]^{H_{0}} \\
+\frac{1}{2}\left\{\nabla_{X} R(Z, Y) u-\nabla_{Y} R(Z, X) u\right\}^{H_{1}}+\frac{1}{2}\left\{\nabla_{X} R(Z, Y) \omega-\nabla_{Y} R(Z, X) \omega\right\}^{H_{2}}
\end{array}\right\},
\end{aligned}
$$




$$
\begin{aligned}
& \text { ii) } \widetilde{R}\left(X^{H_{1}}, Y^{H_{0}}\right) Z^{H_{0}} \\
= & \left\{-\frac{1}{2}\left(\nabla_{Y} R\right)(u, X, Z)\right\}^{H_{0}} \\
& +\left\{\frac{1}{2} R(Z, Y) X-\frac{1}{4} R(R(u, X) Z, Y) u\right\}^{H_{1}}+\left\{-\frac{1}{4} R(R(u, X) Z, Y) \omega\right\}^{H_{2}}
\end{aligned}
$$

iii) $\widetilde{R}\left(X^{H_{0}}, Y^{H_{0}}\right) Z^{H_{1}}$

$$
\begin{aligned}
= & \frac{1}{2}\left\{\left(\nabla_{X} R\right)(u, Z) Y-\left(\nabla_{Y} R\right)(u, Z) X\right\}^{H_{0}} \\
& +\left\{R(X, Y) Z+\frac{1}{4}[R(R(u, Z) Y, X) u-R(R(u, Z) X, Y) u]\right\}^{H_{1}}
\end{aligned}
$$$$
+\frac{1}{4}\{R(R(u, Z) Y, X) \omega-R(R(u, Z) X, Y) \omega\}^{H_{2}},
$$

iv) $\widetilde{R}\left(X^{H_{1}}, Y^{H_{1}}\right) Z^{H_{0}}$

$$
=\left\{R(X, Y) Z+\frac{1}{4}[R(u, X) R(u, Y) Z-R(u, Y) R(u, X) Z]\right\}^{H_{0}},
$$

$$
\begin{aligned}
& v) \widetilde{R}\left(X^{H_{1}}, Y^{H_{0}}\right) Z^{H_{1}} \\
= & \left\{\frac{1}{2} R(X, Z) Y+\frac{1}{4} R(u, X) R(u, Z) Y\right\}^{H_{0}},
\end{aligned}
$$

vi) $\quad \tilde{R}\left(X^{H_{2}}, Y^{H_{0}}\right) Z^{H_{0}}$

$$
\begin{aligned}
= & \left\{-\frac{1}{2}\left(\nabla_{Y} R\right)(\omega, X, Z)\right\}^{H_{0}}+\left\{-\frac{1}{4} R(R(\omega, X) Z, Y) u\right\}^{H_{1}} \\
& +\left\{\frac{1}{2} R(Z, Y) X-\frac{1}{4} R(R(\omega, X) Z, Y) \omega\right\}^{H_{2}},
\end{aligned}
$$

vii) $\quad \widetilde{R}\left(X^{H_{0}}, Y^{H_{0}}\right) Z^{H_{2}}$

$$
\begin{aligned}
= & \frac{1}{2}\left\{\nabla_{X} R(\omega, Z) Y-\nabla_{Y} R(\omega, Z) X\right\}^{H_{0}} \\
& +\frac{1}{4}\{R(R(\omega, Z) Y, X) u-R(R(\omega, Z) X, Y) u\}^{H_{1}} \\
& +\left\{R(X, Y) Z+\frac{1}{4}[R(R(\omega, Z) Y, X) \omega-R(R(\omega, Z) X, Y) \omega]\right\}^{H_{2}},
\end{aligned}
$$

viii) $\quad \widetilde{R}\left(X^{H_{2}}, Y^{H_{2}}\right) Z^{H_{0}}$

$$
=\left\{R(X, Y) Z+\frac{1}{4}[R(\omega, X) R(\omega, Y) Z-R(\omega, Y) R(\omega, X) Z]\right\}^{H_{0}}
$$

ix) $\quad \widetilde{R}\left(X^{H_{2}}, Y^{H_{0}}\right) Z^{H_{2}}$

$$
=\left\{\frac{1}{2} R(X, Z) Y+\frac{1}{4} R(\omega, X) R(\omega, Z) Y\right\}^{H_{0}}
$$




$$
\begin{aligned}
\text { x) } \widetilde{R}\left(X^{H_{1}}, Y^{H_{0}}\right) Z^{H_{2}} & =\left\{\frac{1}{4} R(u, X) R(\omega, Z) Y\right\}^{H_{0}}, \\
\text { xi) } \widetilde{R}\left(X^{H_{1}}, Y^{H_{2}}\right) Z^{H_{0}} & =\frac{1}{4}\{R(u, X) R(\omega, Y) Z-R(\omega, Y) R(u, X) Z\}^{H_{0}}, \\
\text { xii) } \widetilde{R}\left(X^{H_{0}}, Y^{H_{2}}\right) Z^{H_{1}} & =\left\{-\frac{1}{4} R(\omega, Y) R(u, Z) X\right\}^{H_{0}}, \\
\text { xiii) } \widetilde{R}\left(X^{H_{2}}, Y^{H_{0}}\right) Z^{H_{1}} & =\left\{\frac{1}{4} R(\omega, X) R(u, Z) Y\right\}^{H_{0}}, \\
\text { xiv) } \widetilde{R}\left(X^{H_{1}}, Y^{H_{1}}\right) Z^{H_{1}} & =0, \widetilde{R}\left(X^{H_{1}}, Y^{H_{1}}\right) Z^{H_{2}}=0, \widetilde{R}\left(X^{H_{1}}, Y^{H_{2}}\right) Z^{H_{2}}=0, \\
\text { xv) } \widetilde{R}\left(X^{H_{1}}, Y^{H_{2}}\right) Z^{H_{1}} & =0, \widetilde{R}\left(X^{H_{2}}, Y^{H_{2}}\right) Z^{H_{1}}=0, \widetilde{R}\left(X^{H_{2}}, Y^{H_{2}}\right) Z^{H_{2}}=0
\end{aligned}
$$

for all vector fields $X, Y$ on $M$.

Proof. Here we only prove $i$ i). The rest either come immediately from the direct standard calculations or can be proven by following the same method in the proof of $i i$ ). In here, we omit standard calculations and repetation.

Let $F_{1}, F_{2}, F_{3}: T^{2} M \longrightarrow T^{2} M$ be the bundle endomorphisms given by

$$
\begin{aligned}
& F_{1}: u \longrightarrow \frac{1}{2} R(Z, Y) u, \\
& F_{2}: \omega \longrightarrow \frac{1}{2} R(Z, Y) \omega, \\
& F_{3}: u \longrightarrow \frac{1}{2} R(u, X) Z .
\end{aligned}
$$

By using Proposition 3.1, Lemma 3.2 and above equalities, we find

$$
\begin{aligned}
& \widetilde{R}\left(X^{H_{1}}, Y^{H_{0}}\right) Z^{H_{0}} \\
= & \widetilde{\nabla}_{X^{H_{1}}} \widetilde{\nabla}_{Y^{H_{0}}} Z^{H_{0}}-\widetilde{\nabla}_{Y^{H_{0}}} \widetilde{\nabla}_{X^{H_{1}}} Z^{H_{0}}-\widetilde{\nabla}_{\left[X^{H_{1}, Y^{H_{0}}}\right]} Z^{H_{0}} \\
= & \widetilde{\nabla}_{X^{H_{1}}}\left[\left(\nabla_{Y} Z\right)^{H_{0}}+\frac{1}{2}(R(Z, Y) u)^{H_{1}}+\frac{1}{2}(R(Z, Y) \omega)^{H_{2}}\right] \\
& -\widetilde{\nabla}_{Y^{H_{0}}}\left[\frac{1}{2} R(u, X) Z\right]^{H_{0}}+\widetilde{\nabla}_{\left(\nabla_{Y} X\right)^{H_{1}} Z^{H_{0}}} \\
= & \frac{1}{2}\left(R(u, X) \nabla_{Y} Z\right)^{H_{0}}+\left(F_{1}(X)\right)^{H_{1}}-\left(\nabla_{Y} F_{3}(u)\right)^{H_{0}} \\
& -\frac{1}{2}\left(R\left(F_{3}(u), Y\right) u\right)^{H_{1}}-\frac{1}{2}\left(R\left(F_{3}(u), Y\right) \omega\right)^{H_{2}} \\
& +\frac{1}{2}\left(R\left(u, \nabla_{Y} X\right) Z\right)^{H_{0}} \\
& \frac{1}{2}\left\{R(u, X) \nabla_{Y} Z+R\left(u, \nabla_{Y} X\right) Z-\nabla_{Y} R(u, X) Z\right\}^{H_{0}} \\
& +\left\{\frac{1}{2} R(Z, Y) X-\frac{1}{4} R(R(u, X) Z, Y) u\right\} \\
& \left.+\left\{-\frac{1}{4} R(R(u, X) Z), Y\right) \omega\right\}^{H_{1}} \\
& \left.\left.+\left\{-\frac{1}{4} R(R(u, X) Z), Y\right) \omega\right\}^{H_{2}} \cdot \begin{array}{c}
\frac{1}{2} R(Z, Y) X \\
-\frac{1}{4} R(R(u, X) Z, Y) u
\end{array}\right\}
\end{aligned}
$$


For the sectional curvature tensor field $\widetilde{K}$ of $\left(T^{2} M, \widetilde{g}\right)$, we have the following.

Proposition 3.4. Let $(M, g)$ be a Riemannian manifold and $T^{2} M$ be its second-order tangent bundle equipped with the Sasaki metric $\widetilde{g}$. The sectional curvature tensor field $\widetilde{K}$ on $T^{2} M$ satisfies the following:

$$
\begin{aligned}
\text { i) } \widetilde{K}\left(X^{H_{0}}, Y^{H_{0}}\right) & =K(X, Y)-\frac{3}{4}\left(|R(X, Y) u|^{2}+|R(X, Y) \omega|^{2}\right), \\
\text { ii) } \widetilde{K}\left(X^{H_{1}}, Y^{H_{0}}\right) & =\frac{1}{4}|R(u, X) Y|^{2}, \\
\text { iii) } \widetilde{K}\left(X^{H_{2}}, Y^{H_{0}}\right) & =\frac{1}{4}|R(\omega, X) Y|^{2} \\
\text { iv) } \widetilde{K}\left(X^{H_{1}}, Y^{H_{1}}\right) & =0, \widetilde{K}\left(X^{H_{1}}, Y^{H_{2}}\right)=0, \widetilde{K}\left(X^{H_{2}}, Y^{H_{2}}\right)=0
\end{aligned}
$$

for any orthonormal vector fields $X$ and $Y$ on $(M, g)$, where $|$.$| denotes the norm with respect to the Riemannian metric$ $g$.

Proof. Let $\widetilde{K}(\widetilde{X}, \widetilde{Y})$ be the sectional curvature tensor field of the 2-dimensional section generated by orthonormal vectors $\widetilde{X}, \widetilde{Y}$, that is:

$$
\widetilde{K}(\widetilde{X}, \widetilde{Y})=\widetilde{g}((\widetilde{R}(\widetilde{X}, \widetilde{Y}) \widetilde{Y}, \widetilde{X})
$$

i) If $\widetilde{X}=X^{H_{0}}, \widetilde{Y}=Y^{H_{0}}$, then

$$
\begin{aligned}
& \widetilde{K}\left(X^{H_{0}}, Y^{H_{0}}\right) \\
= & \widetilde{g}\left(\widetilde{R}\left(X^{H_{0}}, Y^{H_{0}}\right) Y^{H_{0}}, X^{H_{0}}\right) \\
= & \widetilde{g}\left(\left[R(X, Y) Y+\frac{-3}{4} R(u, R(Y, X) u) Y+\frac{-3}{4} R(\omega, R(Y, X) \omega) Y\right]^{H_{0}}, X^{H_{0}}\right) \\
= & g\left(R(X, Y) Y+\frac{-3}{4} R(u, R(Y, X) u) Y+\frac{-3}{4} R(\omega, R(Y, X) \omega) Y, X\right) \\
= & g(R(X, Y) Y, X)-\frac{3}{4} g(R(u, R(Y, X) u) Y, X)-\frac{3}{4} g(R(\omega, R(Y, X) \omega) Y, X) \\
= & K(X, Y)-\frac{3}{4}\left(|R(X, Y) u|^{2}+|R(X, Y) \omega|^{2}\right),
\end{aligned}
$$

where $K(X, Y)$ is the sectional curvature tensor field on $M$.

ii) If $\widetilde{X}=X^{H_{1}}, \widetilde{Y}=Y^{H_{0}}$, then

$$
\begin{aligned}
\widetilde{K}\left(X^{H_{1}}, Y^{H_{0}}\right) & =\widetilde{g}\left(\widetilde{R}\left(X^{H_{1}}, Y^{H_{0}}\right) Y^{H_{0}}, X^{H_{1}}\right) \\
& =\widetilde{g}\left(\left[-\frac{1}{4} R(R(u, X) Y, Y) u\right]^{H_{1}}, X^{H_{1}}\right) \\
& =-\frac{1}{4} g(R(R(u, X) Y, Y) u, X)=\frac{1}{4}|R(u, X) Y|^{2} .
\end{aligned}
$$

iii) If $\tilde{X}=X^{H_{2}}, \widetilde{Y}=Y^{H_{0}}$, then

$$
\begin{aligned}
& \widetilde{K}\left(X^{H_{2}}, Y^{H_{0}}\right) \\
= & \widetilde{g}\left(\widetilde{R}\left(X^{H_{2}}, Y^{H_{0}}\right) Y^{H_{0}}, X^{H_{2}}\right) \\
= & \widetilde{g}\left(\left[-\frac{1}{4} R(R(\omega, X) Y, Y) \omega\right]^{H_{2}}, X^{H_{2}}\right) \\
= & -\frac{1}{4} g(R(R(\omega, X) Y, Y) \omega, X)=\frac{1}{4}|R(\omega, X) Y|^{2} .
\end{aligned}
$$

Other cases being zero. In here we used the definition of the Sasaki metric $\widetilde{g}$ and Proposition 3.3. 
Proposition 3.5. Let $(M, g)$ be a Riemannian manifold and $T^{2} M$ be its second-order tangent bundle equipped with the Sasaki metric $\widetilde{g}$. Let $S$ be scalar curvarture of $g$ and $\widetilde{S}$ be scalar curvature of $\widetilde{g}$. Then the following equation holds

$$
\widetilde{S}=S-\frac{1}{4} \sum_{i, j=1}^{m}\left(\left|R\left(X_{i}, X_{j}\right) u\right|^{2}+\left|R\left(X_{i}, X_{j}\right) \omega\right|^{2}\right),
$$

where $\left\{X_{1}, \ldots, X_{m}\right\}$ is a local orthonormal frame for $M$.

Proof. Let $\left\{X_{1}, \ldots, X_{m}\right\}$ be an orthonormal basis for the tangent space $T_{p} M$ at the point $p$. Then we can define lifts by $X_{i}{ }^{H_{0}}=Y_{i}, X_{i}{ }^{H_{1}}=Y_{m+i}$, and $X_{i}{ }^{H_{2}}=Y_{2 m+i}$. In the case, the set $\left\{Y_{1}, Y_{2}, \ldots, Y_{3 m}\right\}$ is an orthonormal basis for the tangent space $T_{(p, u, \omega)} T^{2} M$ with respect to the Sasaki metric $\widetilde{g}$. Proposition 3.4 immediately gives

$$
\begin{aligned}
& \widetilde{S}=\sum_{i, j=1}^{3 m} \widetilde{K}\left(Y_{i}, Y_{j}\right) \\
& =\sum_{i, j=1}^{3 m} \widetilde{K}\left(X_{i}{ }^{H_{0}}, X_{j}{ }^{H_{0}}\right)+\widetilde{K}\left(X_{i}^{{ }^{H_{1}}}, X_{j}{ }^{H_{1}}\right) \\
& +\widetilde{K}\left(X_{i}{ }^{H_{2}}, X_{j}{ }^{H_{2}}\right)+2 \widetilde{K}\left(X_{i}{ }^{H_{0}}, X_{j}{ }^{H_{1}}\right)+2 \widetilde{K}\left(X_{i}{ }^{H_{0}}, X_{j}{ }^{H_{2}}\right) \\
& +2 \widetilde{K}\left(X_{i}^{H_{1}}, X_{j}{ }^{H_{2}}\right) \\
& =\sum_{i, j=1}^{3 m}\left(K\left(X_{i}, X_{j}\right)-\frac{3}{4}\left|R\left(X_{i}, X_{j}\right) u\right|^{2}-\frac{3}{4}\left|R\left(X_{i}, X_{j}\right) \omega\right|^{2}\right) \\
& +2\left(\frac{1}{4}\left|R\left(u, X_{j}\right) X_{i}\right|^{2}\right)+2\left(\frac{1}{4}\left|R\left(\omega, X_{j}\right) X_{i}\right|^{2}\right)
\end{aligned}
$$

from which we get, using $\left|R\left(u, X_{j}\right) X_{i}\right|^{2}=\left|R\left(X_{i}, X_{j}\right) u\right|^{2}$,

$$
\widetilde{S}=S-\frac{1}{4} \sum_{i, j=1}^{3 m}\left(\left|R\left(X_{i}, X_{j}\right) u\right|^{2}+\left|R\left(X_{i}, X_{j}\right) \omega\right|^{2}\right) .
$$

Let us assume that $(M, g)$ be an $m$-dimensional Riemannian manifold of constant sectional curvature $\kappa$, that is:

$$
R(X, Y) Z=\kappa(g(Y, Z) X-g(X, Z) Y)
$$

and the scalar curvature $S$ is given by $S=\kappa m(m-1)$.

Corollary 3.6. Let $(M, g)$ be a Riemannian manifold of constant sectional curvature $\kappa$ and $T^{2} M$ be its second-order tangent bundle equipped with the Sasaki metric $\widetilde{g}$. Then

$$
\begin{aligned}
\widetilde{K}\left(X^{H_{0}}, Y^{H_{0}}\right)= & \kappa-\frac{3}{4} \kappa^{2}\left(g(u, Y)^{2}+g(X, u)^{2}\right) \\
& -\frac{3}{4} \kappa^{2}\left(g(\omega, Y)^{2}+g(X, \omega)^{2}\right), \\
\widetilde{K}\left(X^{H_{1}}, Y^{H_{0}}\right)= & \frac{1}{4} \kappa^{2} g(u, Y)^{2}, \\
\widetilde{K}\left(X^{H_{2}}, Y^{H_{0}}\right)= & \frac{1}{4} \kappa^{2} g(\omega, Y)^{2}
\end{aligned}
$$

for any orthonormal vector fields $X, Y$ on $T^{2} M$.

Corollary 3.7. Let $(M, g)$ be a Riemannian manifold of constant sectional curvature $\kappa$ and $T^{2} M$ be its second-order tangent bundle equipped with the Sasaki metric $\widetilde{g}$. Then, the scalar curvature $\widetilde{S}$ is given by

$$
\widetilde{S}=\kappa m(m-1)-\frac{1}{2} \kappa^{2}(m-1)|u|^{2}-\frac{1}{2} \kappa^{2}(m-1)|\omega|^{2} .
$$




\section{Weakly symmetry properties of the Sasaki metric}

A Riemannian manifold $(M, g)$ is called weakly symmetric if there exist two 1 -forms $\alpha_{1}, \alpha_{2}$ and a vector field $A$ on $M$, such that: [4]

$$
\begin{aligned}
& \left(\nabla_{W} R\right)(X, Y, Z) \\
= & \alpha_{1}(W) R(X, Y) Z+\alpha_{2}(X) R(W, Y) Z+\alpha_{2}(Y) R(X, W) Z \\
& +\alpha_{2}(Z) R(X, Y) W+g(R(X, Y) Z, W) A,
\end{aligned}
$$

where $A=\left(\alpha_{2}\right)^{\#}$ and $\alpha_{i} g^{i j}=\alpha^{j}=\alpha^{\#}$, that is, $A$ is the $g$-dual vector field of the 1 -form $\alpha_{2}$. In [1], Bejan and Crasmarenu proved that the weakly symmetry property of the Sasaki metric on the tangent bundle is equivalent to the flatness of the base manifold, generalizing the result obtained in [2] by Binh and Tamassy. In this section we consider the result to extend for $\left(T^{2} M, \widetilde{g}\right)$.

Theorem 4.1. Let $(M, g)$ be a Riemannian manifold and $T^{2} M$ be its second-order tangent bundle equipped with the Sasaki metric $\widetilde{g} .\left(T^{2} M, \widetilde{g}\right)$ is weakly symmetric if and only if $(M, g)$ is flat, and hence $\left(T^{2} M, \tilde{g}\right)$ must be flat too.

Proof. In the proof, we follow the method used in [1] using Proposition 3.3. In view of Proposition 3.3, $R=0$ immediately gives $\widetilde{R}=0$ and so we have (4.1) as null equality. Firstly, consider the condition (4.1) for $W^{H_{0}}, X^{H_{0}}, Y^{H_{2}}$ and $Z^{H_{2}}$ and we find

$$
\begin{aligned}
& \alpha_{1}\left(W^{H_{0}}\right) \tilde{R}\left(X^{H_{0}}, Y^{H_{2}}\right) Z^{H_{2}}+\alpha_{2}\left(X^{H_{0}}\right) \tilde{R}\left(W^{H_{0}}, Y^{H_{2}}\right) Z^{H_{2}} \\
& +\alpha_{2}\left(Y^{H_{2}}\right) \tilde{R}\left(X^{H_{0}}, W^{H_{0}}\right) Z^{H_{2}}+\alpha_{2}\left(Z^{H_{2}}\right) \tilde{R}\left(X^{H_{0}}, Y^{H_{2}}\right) W^{H_{0}} \\
& +\tilde{g}\left(\tilde{R}\left(X^{H_{0}}, Y^{H_{2}}\right) Z^{H_{2}}, W^{H_{0}}\right)\left(a_{2}\right)^{\#} \\
= & -\nabla_{W^{H_{0}}}\left[-\frac{1}{2} R(Y, Z) X-\frac{1}{4} R(\omega, Y) R(\omega, Z) X\right]^{H_{0}} \\
& -\tilde{R}\left(\left(\nabla_{W} X\right)^{H_{0}}+\left(\frac{1}{2} R(X, W) u\right)^{H_{1}}+\left(\frac{1}{2} R(X, W) \omega\right)^{H_{2}}, Y^{H_{2}}\right) Z^{H_{2}} \\
& -\tilde{R}\left(X^{H_{0}},\left(\frac{1}{2} R(\omega, Y) W\right)^{H_{0}}+\left(\nabla_{W} Y\right)^{H_{2}}\right) Z^{H_{2}} \\
& -\tilde{R}\left(X^{H_{0}}, Y^{H_{2}}\right)\left(\frac{1}{2}(R(\omega, Z) W)^{H_{0}}+\left(\nabla_{W} Z\right)^{H_{2}}\right)
\end{aligned}
$$

Secondly, consider the $H_{2}$ part of the two sides of the above equation, we obtain

$$
\begin{aligned}
& \alpha_{2}\left(Y^{H_{2}}\right)\left(R(X, W) Z+\frac{1}{4} R(R(\omega, Z) W, X) \omega-\frac{1}{4} R(R(\omega, Z) X, W) \omega\right) \\
& +\alpha_{2}\left(Z^{H_{2}}\right)\left(-\frac{1}{2} R(W, X) Y+\frac{1}{4} R(R(\omega, Y) W, X) \omega\right) \\
& -\tilde{g}\left(\frac{1}{2} R(Y, Z) X+\frac{1}{4} R(\omega, Y)(\omega, Z) X, W\right) \alpha_{2}^{\#} \\
& =\frac{1}{4} R(R(Y, Z) X, W) \omega+\frac{1}{8} R(R(\omega, Y) R(\omega, Z) X, W) \omega \\
& -\frac{1}{2}\left(\begin{array}{c}
\left.R(X, R(\omega, Y) W) Z+\frac{1}{4} R(R(\omega, Z) R(\omega, Y) W, X) \omega\right) \\
-R(R(\omega, Z) X, R(\omega, Y) W) \omega
\end{array}\right) \\
& -\frac{1}{2}\left(-\frac{1}{2} R(R(\omega, Z) W, X) Y+\frac{1}{4} R(R(\omega, Y) R(\omega, Z) W, X) \omega\right)
\end{aligned}
$$


By setting $Y=\omega$ and $Z=\omega$ respectively we get

$$
\begin{aligned}
& \alpha_{2}\left(\omega^{H_{2}}\right)\left(R(X, W) Z+\frac{1}{4}(R(R(\omega, Z) W, X) \omega-R(R(\omega, Z) X, W) \omega)\right) \\
& +\alpha_{2}\left(Z^{H_{2}}\right)\left(-\frac{1}{2} R(W, X) \omega\right)-\tilde{g}\left(\frac{1}{2} R(\omega, Z) X, W\right) \alpha_{2}^{\#} \\
= & \frac{1}{4} R(R(\omega, Z) X, W) \omega+\frac{1}{4} R(R(\omega, Z) W, X) \omega
\end{aligned}
$$

and

$$
\begin{aligned}
& \alpha_{2}\left(\omega^{H_{2}}\right)\left(-\frac{1}{2} R(W, X) Y+\frac{1}{4} R(R(\omega, Y) W, X) \omega\right) \\
& +\alpha_{2}\left(Y^{H_{2}}\right)(R(X, W) \omega)-\tilde{g}\left(\frac{1}{2} R(Y, \omega) X, W\right) \alpha_{2}^{\#} \\
& =\frac{1}{4} R(R(Y, \omega) X, W) \omega-\frac{1}{2} R(X, R(\omega, Y) W) \omega .
\end{aligned}
$$

On replacing $Y$ by $Z$ in (4.5), then

$$
\begin{aligned}
& \alpha_{2}\left(\omega^{H_{2}}\right)\left(-\frac{1}{2} R(W, X) Z+\frac{1}{4} R(R(\omega, Z) W, X) \omega\right) \\
& +\alpha_{2}\left(Z^{H_{2}}\right) R(X, W) \omega-\tilde{g}\left(\frac{1}{2} R(Z, \omega) X, W\right) \alpha_{2}{ }^{\#} \\
& =\frac{1}{4} R(R(Z, \omega) X, W) \omega-\frac{1}{2} R(X, R(\omega, Z) W) \omega
\end{aligned}
$$

and by summing (4.4) and (4.6) we get

$$
\begin{aligned}
& \frac{3}{2} \alpha_{2}\left(Z^{H_{2}}\right) R(X, W) \omega \\
& +\alpha_{2}\left(\omega^{H_{2}}\right)\left(\frac{3}{2} R(X, W) Z+\frac{1}{2} R(R(\omega, Z) W, X) \omega-\frac{1}{4} R(R(\omega, Z) X, W) \omega\right) \\
= & -\frac{3}{4} R(X, R(\omega, Z) W) \omega .
\end{aligned}
$$

The equation (4.7) with $Z=\omega$ reduces to

$$
\alpha_{2}\left(\omega^{H_{2}}\right) R(X, W) \omega=0 .
$$

If $\alpha_{2}\left(\omega^{H_{2}}\right) \neq 0$, then the result follows. Suppose now that $\alpha_{2}\left(\omega^{H_{2}}\right)=0$ then $\left(\left(\alpha_{2}\right)^{\#}\right)^{H_{2}}=0$. Returning to (4.4) it results

$$
\alpha_{2}\left(Z^{H_{2}}\right)\left(-\frac{1}{2} R(W, X) \omega\right)=\frac{1}{4} R(R(\omega, Z) X, W) \omega+\frac{1}{4} R(R(\omega, Z) W, X) \omega
$$

from which, by exchanging $W$ and $X$, we obtain

$$
R(R(\omega, Z) X, X) \omega=0
$$

and when we take the inner product with $Z$, it follows that

$$
g(R(\omega, Z) X, R(\omega, Z) X)=0 .
$$

Thus,

$$
R(\omega, Z) X=0 .
$$

Again the $g$-product with an arbitrary $Y$ gives:

$$
g(R(X, Y) \omega, Z)=0 .
$$

For $Z$ being an arbitrary vector field we have $R(X, Y) \omega=0$, for every $X, Y$ and $\omega$. So we have $R=0$ which completes proof. 


\section{Statistical structures on the second-order tangent bundle}

Let $\nabla$ be an arbitrary linear connection on a (pseudo-)Riemannian manifold $(M, g)$. Given the pair $(\nabla, g)$, we construct the $(0,3)$-tensor field $F$ by

$$
F(X, Y, Z):=\left(\nabla_{Z} g\right)(X, Y)
$$

for any vector fields $X, Y, Z$ on $M$. The tensor field $F$ is sometimes referred to as the cubic form associated to the pair $(\nabla, g)$.

For a symmetric bilinear form $\rho$ on a manifold $M$, we call $(\nabla, \rho)$ a Codazzi pair, if its covariant derivative $(\nabla \rho)$ is totally symmetric in $X, Y, Z:[14]$

$$
\left(\nabla_{X} \rho\right)(Y, Z)=\left(\nabla_{Y} \rho\right)(X, Z)=\left(\nabla_{Z} \rho\right)(X, Y) .
$$

In terms of the cubic form $F$, this condition is stated

$$
F(X, Y, Z)=F(Z, Y, X)=F(Z, X, Y)
$$

i.e., the condition $(\nabla, g)$ being Codazzi pair is equivalent to $F$ being totally symmetric in all of its indices. Now, we search the conditions under which the pair $\left(\nabla^{h}, \widetilde{g}\right)$ is a Codazzi pair on $T^{2} M$. We first define the horizontal lift of the torsion-free linear connection $\nabla$ on $M$. The horizontal lift $\nabla^{h}$ of $\nabla$ to $T^{2} M$ is a unique classical linear connection on $T^{2} M$ satisfying

$$
\nabla_{X^{H_{0}}}^{h} Y^{H_{b}}=\left(\nabla_{X} Y\right)^{H_{b}}, \nabla_{X^{H_{a}}}^{h} Y^{H_{b}}=0
$$

for all vector fields $X, Y$ on $M$, where $a=1,2, b=0,1,2$ [6].

Theorem 5.1. Let $\nabla$ be an arbitrary linear connection on a Riemannian manifold $(M, g)$ and $T^{2} M$ be its second-order tangent bundle with the Sasaki metric $\widetilde{g}$. The pair $\left(\nabla^{h}, \widetilde{g}\right)$ is a Codazzi pair on $T^{2} M$ if and only if $\nabla$ is a metric connection with respect to $g$.

Proof. Firstly we compute the covariant derivative of the Sasaki metric $\widetilde{g}$ with respect to the horizontal lift connection $\nabla^{h}$ : for all vector fields $X, Y, Z$ on $M$,

$$
\left\{\begin{array}{c}
\left(\nabla_{X^{H_{0}}}^{h} \widetilde{g}\right)\left(Y^{H_{a}}, Z^{H_{b}}\right)=\left(\nabla_{X} g\right)(Y, Z), \text { for } a=b=0,1,2 \\
\left(\nabla_{X}^{h} \widetilde{g}\right)\left(Y^{H_{a}}, Z^{H_{b}}\right)=0, \text { for } a \neq b \\
\left(\nabla_{X^{H_{c}}}^{h} \widetilde{g}\right)\left(Y^{H_{a}}, Z^{H_{b}}\right)=0, \text { for } a, b=0,1,2 \text { and } c=1,2 .
\end{array}\right.
$$

For all vector fields $\widetilde{X}, \widetilde{Y}, \widetilde{Z}$ on $T^{2} M$, the Codazzi equation on $T^{2} M$ with the Sasaki metric with respect to the horizontal lift connection $\nabla^{h}$ is given by

$$
\left(\nabla_{\widetilde{X}}^{h} \widetilde{g}\right)(\widetilde{Y}, \widetilde{Z})=\left(\nabla_{\widetilde{Y}}^{h} \widetilde{g}\right)(\widetilde{X}, \widetilde{Z})=\left(\nabla_{\widetilde{Z}}^{h} \widetilde{g}\right)(\widetilde{X}, \widetilde{Y}) .
$$

If $\widetilde{X}=X^{H_{0}}, \tilde{Y}=Y^{H_{1}}, \widetilde{Z}=Z^{H_{1}}$, then

$$
\begin{aligned}
\left(\nabla_{X^{H_{0}}}^{h} \widetilde{g}\right)\left(Y^{H_{1}}, Z^{H_{1}}\right) & =\left(\nabla_{Y^{H_{1}}}^{h} \widetilde{g}\right)\left(X^{H_{0}}, Z^{H_{1}}\right) \\
\left(\nabla_{X} g\right)(Y, Z) & =0 .
\end{aligned}
$$

In the case, from (5.3), we can say that the pair $\left(\nabla^{h}, \widetilde{g}\right)$ is a Codazzi pair on $T^{2} M$ if and only if $\nabla$ is a metric connection with respect to $g$.

We know, the horizontal lift connection $\nabla^{h}$ of a linear connection $\nabla$ has a torsion. Next we introduce a new connection without torsion so-called a mean connection. With help of the horizontal lift connection and its torsion tensor, the mean connection defined by [13]

$$
\nabla_{\widetilde{X}}^{m} \widetilde{Y}=\nabla_{\widetilde{X}}^{h} \widetilde{Y}-\frac{1}{2} T^{h}(\widetilde{X}, \widetilde{Y})
$$

where $T^{h}$ is the torsion tensor of the horizontal lift connection $\nabla^{h}$. The torsion tensor of $\nabla^{h}$ is given by

$$
\left\{\begin{array}{c}
T^{h}\left(X^{H_{0}}, Y^{H_{0}}\right)=(T(X, Y))^{H_{0}}+(R(X, Y) u)^{H_{1}}+(R(X, Y) \omega)^{H_{2}} \\
T^{h}\left(X^{H_{a}}, Y^{H_{b}}\right)=0, \text { for } a, b=1,2
\end{array}\right.
$$

www.iejgeo.com 
Hence, the mean connection $\nabla^{m}$ satisfies

$$
\begin{aligned}
\nabla_{X}^{H_{0}} Y^{H_{0}} & =\left(\nabla_{X} Y-\frac{1}{2} T(X, Y)\right)^{H_{0}}-\frac{1}{2}(R(X, Y) u)^{H_{1}}-\frac{1}{2}(R(X, Y) \omega)^{H_{2}}, \\
\nabla_{X^{H_{0}}}^{h} Y^{H_{b}} & =\left(\nabla_{X} Y\right)^{H_{b}}, \text { for } b=1,2 \\
\nabla_{X^{H_{a}}}^{m} Y^{H_{b}} & =0 \text {, for } a, b=1,2 .
\end{aligned}
$$

In the rest of the paper, we consider a torsion-free linear connection $\nabla$ on a pseudo-Riemannian manifold $(M, g)$. In the case, if $(\nabla, g)$ is a Codazzi pair which characterizes what is known to information geometers as statistical structures, then the manifold $M$ together with a statistical structure $(\nabla, g)$ is called a statistical manifold. The notion of statistical manifold was originally introduced by Lauritzen [11]. Statistical manifolds are widely studied in affine differential geometry [11,12] and plays a central role in information geometry.

As is known, the torsion tensor of the horizontal lift connection $\nabla^{h}$ of a linear connection $\nabla$ is zero if and only if the connection $\nabla$ is flat. Thus, from Theorem 5.1, we immediately give the following result.

Corollary 5.2. Let $\nabla$ be the Levi-Civita conection on a Riemannian manifold $(M, g)$ and $T^{2} M$ be its second-order tangent bundle with the Sasaki metric $\widetilde{g}$. The pair $\left(\nabla^{h}, \widetilde{g}\right)$ is a statistical structure on $T^{2} M$ if and only if $(M, g)$ is flat.

Theorem 5.3. Let $\nabla$ be an arbitrary linear connection on a Riemannian manifold $(M, g)$ and $T^{2} M$ be its secondorder tangent bundle with the Sasaki metric $\widetilde{g}$. The pair $\left(\nabla^{m}, \widetilde{g}\right)$ is a statistical structure on $T^{2} M$ if and only if $\nabla$ is a metric connection with respect to $g$, its Riemannian curvature tensor is zero and its torsion tensor satisfies $2 g(T(X, Y), Z)=g(X, T(Y, Z))-g(Y, T(X, Z))$.

Proof. Applying the covariant derivation operator $\nabla^{m}$ to the Sasaki metric $\widetilde{g}$, we find

$$
\left\{\begin{array}{c}
\left(\nabla_{X^{H_{0}}}^{m} \widetilde{g}\right)\left(Y^{H_{0}}, Z^{H_{0}}\right)=\left(\nabla_{X} g\right)(Y, Z) \\
+\frac{1}{2} g(T(X, Y), Z)+\frac{1}{2} g(Y, T(X, Z)), \\
\left.\nabla_{X^{H_{0}}}^{m} \widetilde{g}\right)\left(Y^{H_{a}}, Z^{H_{b}}\right)=\left(\nabla_{X} g\right)(Y, Z), \text { for } a=b=1,2 \\
\left(\nabla_{X^{H_{0}}}^{H_{0}} \widetilde{g}\right)\left(Y^{H_{0}}, Z^{H_{1}}\right)=\frac{1}{2} g(R(X, Y) u, Z), \\
\left(\nabla_{X^{H_{0}}}^{H_{0}}\right)\left(Y^{H_{0}}, Z^{H_{2}}\right)=\frac{1}{2} g(R(X, Y) \omega, Z), \\
\left(\nabla_{X_{0} H_{0}}^{H_{0}}\right)\left(Y^{H_{a}}, Z^{H_{b}}\right)=0, \text { for } a \neq b=1,2 \\
\left(\nabla_{X^{H_{c}}}^{m} \widetilde{g}\right)\left(Y^{H_{a}}, Z^{H_{b}}\right)=0, \text { for } a, b=0,1,2 \text { and } c=1,2 .
\end{array}\right.
$$

for all vector fields $X, Y, Z$ on $M$.

If $\widetilde{X}=X^{H_{0}}, \widetilde{Y}=Y^{H_{1}}, \widetilde{Z}=Z^{H_{1}}$, then

$$
\begin{aligned}
\left(\nabla_{X^{H_{0}}}^{h} \widetilde{g}\right)\left(Y^{H_{1}}, Z^{H_{1}}\right) & =\left(\nabla_{Y^{H_{1}}}^{h} \widetilde{g}\right)\left(X^{H_{0}}, Z^{H_{1}}\right) \\
\left(\nabla_{X} g\right)(Y, Z) & =0 .
\end{aligned}
$$

If $\widetilde{X}=X^{H_{0}}, \widetilde{Y}=Y^{H_{0}}, \widetilde{Z}=Z^{H_{1}}$, then

$$
\begin{aligned}
\left(\nabla_{X^{H_{0}}}^{h} \widetilde{g}\right)\left(Y^{H_{0}}, Z^{H_{1}}\right) & =\left(\nabla_{Z^{H_{1}}}^{h} \widetilde{g}\right)\left(X^{H_{0}}, Y^{H_{0}}\right) \\
g(R(X, Y) u, Z) & =0 .
\end{aligned}
$$

If $\widetilde{X}=X^{H_{0}}, \widetilde{Y}=Y^{H_{0}}, \widetilde{Z}=Z^{H_{0}}$, using (5.4), we derive

$$
\begin{aligned}
\left(\nabla_{X}^{h}{ }_{H_{0}} \widetilde{g}\right)\left(Y^{H_{0}}, Z^{H_{0}}\right) & =\left(\nabla_{Y H_{0}}^{h} \widetilde{g}\right)\left(X^{H_{0}}, Z^{H_{0}}\right) \\
\frac{1}{2} g(T(X, Y), Z)+\frac{1}{2} g(Y, T(X, Z)) & =\frac{1}{2} g(T(Y, X), Z)+\frac{1}{2} g(X, T(Y, Z)) \\
2 g(T(X, Y), Z) & =g(X, T(Y, Z))-g(Y, T(X, Z)) .
\end{aligned}
$$

Therefore, (5.4)- (5.6) give the result.

\section{References}

[1] Bejan, C. L., Crasmareanu, M.: Weakly-symmetry of the Sasakian lifts on tangent bundles, Publ. Math. Debrecen, 83 (1-2), $63-69$ (2013).

[2] Binh, T. Q., Tamassy, L.: On recurrence or pseudo-symmetry of the Sasakian metric on the tangent bundle of a Riemannian manifold, Indian J. Pure Appl. Math., 35 ( 4), 555-560 (2004). 
[3] de Leon, M., Vazquez, E.: On the geometry of the tangent bundle of order 2, An. Univ. Bucureşti Mat., 34, 40-48 (1985).

[4] De, U. C., Bandyopadhyay, S.: On weakly symmetric Riemannian spaces, Publ. Math. Debrecen, 54 (3-4), 377-381 (1999).

[5] Dida, M. H., Hathout, F., Djaa, M.: On the geometry of the second order tangent bundle with the diagonal lift metric, Int. J. Math. Anal., 3 (9-12), 443-456 (2009).

[6] Djaa, M., Gancarzewicz, J.: The geometry of tangent bundles of order $r$, Boletin Academia, Galega de Ciencias, 4, 147-165 (1985).

[7] Dodson, C. T. J., Radivoiovici, M. S.: Tangent and frame bundles of order two, Analele stiintifice ale Universitatii "Al. I. Cuza", 28, 63-71 (1982).

[8] Gezer, A., Magden, A.: Geometry of the second-order tangent bundles of Riemannian manifolds, Chin. Ann. Math. Ser. B, 38 (4), $985-998$ (2017).

[9] Hathout, F, Dida, H. M.: Diagonal lift in the tangent bundle of order two and its applications, Turkish J. Math., 30 (4), $373-384$ (2004).

[10] Ishikawa, S.: On Riemannian metrics of tangent bundles of order 2 of Riemannian manifolds, Tensor (N.S.), 34 (2), 173-178 (1980).

[11] Lauritzen, S. L.: Statistical manifolds( In: Differential Geometry in Statistical Inferences, IMS Lecture Notes Monogr. Ser., 10, Inst. Math. Statist., Hayward California, 1987, 96- 163).

[12] Nomizu, K., Sasaki, T.: Affine Differential Geometry Geometry of Affine Immersions, vol. 111 of Cambridge Tracts in Mathematics. Cambridge University Press, Cambridge 1994.

[13] Oniciuc, C.: The tangent bundles and harmonicity, An. Ştiinț. Univ. Al. I. Cuza Iaşi. Mat. (N.S.), 43 (1), 151-172 (1987)

[14] Schwenk-Schellschmidt, A., Simon, U.: Codazzi-equivalent affine connections, Result Math., 56 (1-4), 211-229 (2009).

[15] Yano, K., Ishihara, S.: Tangent and cotangent bundles, Marcel Dekker, Inc., New York, 1973.

\section{Affiliations}

ABDUlLah MAGDEN

AdDrEss: Bursa Technical University, Dept. of Mathematics, Bursa-Turkey.

E-MAIL: abdullah.magden@btu.edu.tr

ORCID ID:0000-0002-9513-7012

AYDIN GEZER

ADDRESS: Ataturk University, Dept. of Mathematics, 25240, Erzurum-Turkey.

E-MAIL: aydingzr@gmail.com

ORCID ID:0000-0001-7505-0385

KUBRA KARACA

ADDRESS: Ataturk University, Dept. of Mathematics, 25240, Erzurum-Turkey.

E-MAIL: kubrakaraca91@gmail.com

ORCID ID:0000-0002-6329-4435 